\title{
A road towards a beyond the Standard Model model
}

\author{
Giancarlo Rossi ${ }^{1,2,3, *}$ \\ ${ }^{1}$ Università di Roma Tor Vergata, Via della Ricerca Scientifica - 00133 Roma - Italy \\ ${ }^{2}$ INFN - Sezione di Roma Tor Vergata, Via della Ricerca Scientifica - 00133 Roma - Italy \\ ${ }^{3}$ Centro Fermi - Piazza del Viminale, 1 - 00184 Roma - Italy
}

\begin{abstract}
In this talk we describe examples of renormalizable strongly interacting field theories where chiral symmetry, broken at the UV cutoff by the presence of some irrelevant $d>4$ operators in the fundamental Lagrangian, is recovered at low energy owing to the tuning of certain Lagrangian parameters. The interference of UV effects with IR features coming from the spontaneous breaking of the recovered chiral symmetry yields non perturbatively generated elementary fermion masses parametrically expressed by formulae of the kind $m_{q} \sim C_{q}(\alpha) \Lambda_{\mathrm{RGI}}$ with $\alpha$ the gauge coupling constant and $\Lambda_{\mathrm{RGI}}$ the RGI scale of the theory. Upon introducing EW interactions, this mechanism can be extended to give mass to EW bosons and leptons and can thus be used as an alternative to the Higgs scenario. In order to give the top quark and the weak gauge bosons a mass of the phenomenologically correct order of magnitude, the model must necessarily include (yet unobserved) super-strongly interacting massive fermions endowed, besides ordinary Standard Model interactions, with super-strong interactions with a RGI scale, $\Lambda_{T} \gg \Lambda_{Q C D}$ in the few TeV range. Though limited in its scope (here we ignore hypercharge and leptons and discuss only the case of one family neglecting weak isospin splitting), the model opens the way to a solution of the naturalness problem and an understanding of the fermion mass hierarchy.
\end{abstract}

\section{Introduction}

We show in this talk that in strongly interacting field theories where chiral symmetry, broken at the UV cutoff by the presence of some irrelevant $d>4$ operators of the Wilson type in the fundamental Lagrangian, is recovered at low energy owing to the tuning of certain Lagrangian parameters, mass terms for elementary fermions are dynamically generated. They are a consequence of a sort of "interference" between such UV chiral breaking terms and nonperturbative (NP) effects coming from the spontaneous breaking of the (recovered) chiral symmetry occurring in the Nambu-Goldstone (NG) phase of the theory. and are standardly induced by the strong interaction dynamics $[1,2]$. A detailed analysis of this remarkable field-theoretical feature shows that such NP masses have the parametric form

$$
m_{q} \sim C_{q}(\alpha) \Lambda_{\mathrm{RGI}}
$$

where $\Lambda_{\mathrm{RGI}}$ is the RGI scale of the theory and $C_{q}(\alpha)$ is a function of the gauge coupling constants $\{\alpha\}$ of the theory. If we take the irrelevant chiral breaking Wilson-like term to be

\footnotetext{
*e-mail: rossig@roma2.infn.it
} 
a $d=6$ operator, one finds to lowest loop order $C_{q}(\alpha)=\mathrm{O}\left(\alpha_{f}^{2}\right)$, where $\alpha_{f}$ is the strongest among the gauge interactions the particle is subjected to.

An exact symmetry protects elementary masses against power divergencies, at variance with Wilson lattice QCD (WLQCD). Masses are "small", i.e. $\mathrm{O}\left(\Lambda_{\mathrm{RGI}}\right)$, as the massless theory enjoys an enhanced (chiral) symmetry, consistently with the 't Hooft notion of naturalness [3].

A consequence of the formula (1), if applied to the top quark, is that since $m_{\text {top }}=\mathrm{O}\left(\Lambda_{\mathrm{RGI}}\right)$, to match its experimental values a new sector of super-strongly interacting particles, gaugeinvariantly coupled to standard matter, needs to exist so that the complete theory, including the new sector and Standard Model (SM) particles, will have an RGI scale $\Lambda_{\mathrm{RGI}} \equiv \Lambda_{T} \gg$ $\Lambda_{Q C D}$ and of the order of a few TeV's. We immediately notice that the reason for assuming the existence of a super-strongly interacting sector here is very different from the reason why Technicolor was introduced $[4,5]$. Technicolor was invoked to give mass to the EW bosons in the first place, while in the present approach super-strong interactions are introduced to give the right order of magnitude to the top quark and $W$ masses. Anyway to avoid confusion and following Glashow [6], we will refer to this new set of particles as Tera-particles.

The issue of "families" and how eq. (1) can be extended to deal with lighter families (and to cope with weak isospin splitting) is outside the scope of this talk and, as we shall discuss in sect. 4, is related to the question of the universality of the structure of the NP mass formulae.

The model can be naturally extended to incorporate EW interactions and leptons. EW bosons as well as leptons will acquire NP masses $\mathrm{O}\left(\Lambda_{T}\right)$ via the same mechanism that leads to eq. (1) ${ }^{1}$, but with coefficient functions that scale like powers of the EW gauge coupling. For instance, to the leading loop order one gets for the $W$ mass (see sect. 3 and refs. [7, 8])

$$
M_{W} \sim \sqrt{\alpha_{w}} c_{w}(\alpha) \Lambda_{\mathrm{RGI}}, \quad c_{w}(\alpha)=\mathrm{O}(\alpha),
$$

where $\alpha$ in eq. (2) is a short-hand for the set of gauge couplings $\left[\alpha_{s}, \alpha_{T}\right]$ with $\alpha_{s}$ and $\alpha_{T}$ the strong and Tera-strong gauge couplings, respectively.

Eqs. (1) and (2) are somewhat similar to the expression of the Higgs-masses of fermions and $W$ 's with, however, two fundamental differences. The first is that the scale of the mass is not the vev of the Higgs field, but a dynamical scale related to a new interaction. The second is that the modulation of the Yukawa couplings that in the SM is introduced by hand to fit the values of fermion masses, here, as we said before, is controlled to leading order by the magnitude of the gauge coupling of the strongest among the interactions the particle feels.

We conclude from this analysis that the NP scenario for mass generation we advocate here can be considered as a valid alternative to the Higgs mechanism, with the extra advantage that, as we shall see, we will not have to deal with power divergencies in the Higgs mass. A further conceptual bonus is that, in view of the above mass formulae, we have a natural interpretation of the magnitude of the EW scale, as the dynamical physical parameter, $\Lambda_{T}$.

There is a number of further interesting features of the approach we are describing in this talk that are worth mentioning, but which we are not going to develop here for lack of space.

First of all, the dependence of the NP fermion masses upon the gauge couplings $\left(C\left(\alpha_{f}\right)=\right.$ $\mathrm{O}\left(\alpha_{f}^{2}\right)$ to leading order for a $d=6$ Wilson-like operator, see eq. (6)) may offer a hint to understand the fermion mass hierarchy $m_{\tau} \ll m_{t} \ll m_{T}$, as due to the ranking among weak, strong and Tera-strong gauge couplings, $\alpha_{w} \ll \alpha_{s} \ll \alpha_{T}{ }^{2}$.

Secondly, lacking the need for a Higgs boson, we propose to interpret the $125 \mathrm{GeV}$ resonance, recently identified at LHC, as a $W^{+} W^{-} / Z Z$ composite state, bound by exchanges of Tera-particles. Since this bound state is light on the $\Lambda_{T}$ scale, it should be incorporated in the effective low energy theory that one gets by integrating out the Tera-degrees of freedom. As

\footnotetext{
${ }^{1}$ With the SM hypercharge assignment, in our model neutrinos are massless because $v_{R}$ is sterile.

${ }^{2}$ In this talk we limit ourselves to discuss only the heaviest fermion family.
} 
a result, at (momenta) $)^{2} \ll \Lambda_{T}^{2}$ the Quantum Effective Lagrangian (QEL) ${ }^{3}$ of the model can be argued to resemble very much to the SM.

Finally in ref. [9] it has been shown that with a reasonable choice of the elementary particle content, a theory extending the SM with the inclusion of the new super-strong sector leads to gauge coupling unification at $\mathrm{a} \sim 10^{18} \mathrm{GeV}$ scale, yielding a proton life-time comfortably larger than the present limit $\tau_{\text {prot }}>1.7 \times 10^{34}$ years.

\section{A simple toy-model}

The simplest model enjoying the NP mass generation mechanism we outlined before is described by a Lagrangian where an SU(2) fermion doublet, subjected to non-abelian gauge interactions (of the QCD type), is coupled to a complex scalar via $d=4$ Yukawa and "irrelevant" $d=6$ Wilson-like chiral breaking terms. The Lagrangian of this toy-model reads

$$
\begin{aligned}
\mathcal{L}_{\text {toy }}(q, A, \Phi)=\mathcal{L}_{\text {kin }}(q, A, \Phi)+\mathcal{V}(\Phi)+\mathcal{L}_{Y u k}(q, \Phi)+\mathcal{L}_{W i l}(q, A, \Phi) \\
-\mathcal{L}_{\text {kin }}(q, A, \Phi)=\frac{1}{4}\left(F^{A} \cdot F^{A}\right)+\bar{q}_{L} \mathcal{D}^{A} q_{L}+\bar{q}_{R} \mathcal{D}^{A} q_{R}+\frac{1}{2} \operatorname{Tr}\left[\partial_{\mu} \Phi^{\dagger} \partial_{\mu} \Phi\right] \\
\text { - } \mathcal{V}(\Phi)=\frac{\mu_{0}^{2}}{2} \operatorname{Tr}\left[\Phi^{\dagger} \Phi\right]+\frac{\lambda_{0}}{4}\left(\operatorname{Tr}\left[\Phi^{\dagger} \Phi\right]\right)^{2} \\
\text { - } \mathcal{L}_{Y u k}(q, \Phi)=\eta\left(\bar{q}_{L} \Phi q_{R}+\bar{q}_{R} \Phi^{\dagger} q_{L}\right) \\
\text { - } \mathcal{L}_{W i l}(q, A, \Phi)=\frac{b^{2}}{2} \rho\left(\bar{q}_{L} \overleftarrow{\mathcal{D}}_{\mu}^{A} \Phi \mathcal{D}_{\mu}^{A} q_{R}+\bar{q}_{R} \overleftarrow{\mathcal{D}}_{\mu}^{A} \Phi^{\dagger} \mathcal{D}_{\mu}^{A} q_{L}\right),
\end{aligned}
$$

where $\Phi=\varphi_{0} 11+i \varphi_{j} \tau^{j}=\left[-i \tau_{2} \varphi^{\star} \mid \varphi\right]$ with $\varphi=\left(\varphi_{2}-i \varphi_{1}, \varphi_{0}-i \varphi_{3}\right)^{T}$ is a complex scalar doublet, singlet under SU(3), $b^{-1} \sim \Lambda_{U V}$ is the UV cutoff, $\eta$ is the Yukawa coupling, $\rho$ is to keep track of $\mathcal{L}_{W i l}$ and $\mathcal{D}_{\mu}^{A}$ is the covariant derivative. The Lagrangian (3) is power counting renormalizable (like WLQCD is, despite the presence of the $d=5$ Wilson term).

Among other obvious symmetries, $\mathcal{L}_{\text {toy }}$ is invariant under the (global) transformations involving fermions and scalars $\left(\Omega_{L / R} \in \mathrm{SU}(2)\right)$

$$
\begin{aligned}
& \chi_{L} \times \chi_{R}=\left[\tilde{\chi}_{L} \times\left(\Phi \rightarrow \Omega_{L} \Phi\right)\right] \times\left[\tilde{\chi}_{R} \times\left(\Phi \rightarrow \Phi \Omega_{R}^{\dagger}\right)\right], \quad \text { where } \\
& \tilde{\chi}_{L / R}: q_{L / R} \rightarrow \Omega_{L / R} q_{L / R}, \quad \bar{q}_{L / R} \rightarrow \bar{q}_{L / R} \Omega_{L / R}^{\dagger} .
\end{aligned}
$$

The $\chi_{L} \times \chi_{R}$ symmetry can be realized either á la Wigner or á la NG depending on the shape of the scalar potential. In any case no power divergent fermion mass can be generated by quantum corrections as the mass operator $\left(\bar{q}_{L} q_{R}+\bar{q}_{R} q_{L}\right)$ is not invariant under $\chi_{L} \times \chi_{R}$.

The operators $\mathcal{L}_{W i l}$ and $\mathcal{L}_{Y u k}$ break $\tilde{\chi}_{L} \times \tilde{\chi}_{R}$ and mix. Thus for generic values of $\eta, \mathcal{L}_{\text {toy }}$ is not invariant under the fermionic chiral transformations $\tilde{\chi}_{L} \times \tilde{\chi}_{R}$. However, one can show [1, 10] that chiral invariance can be recovered (up to vanishingly small $\mathrm{O}\left(b^{2}\right)$ terms) at a critical value $\eta=\eta_{c r}$, where the Wilson-like term and the Yukawa terms "compensate", much like chiral symmetry is recovered (up to $\mathrm{O}(a)$ cutoff effects) in WLQCD by tuning the bare quark mass to a critical value, $m_{c r}$, where the Wilson and the mass term "compensate".

In the Wigner phase (where $\left\langle|\Phi|^{2}\right\rangle=0$ ) the condition determining $\eta_{c r}$ corresponds to the vanishing of the effective Yukawa term in the QEL of the theory. Diagrammatically we represent in fig. 1 this condition to lowest loop order.

In the NG phase (where $\left\langle|\Phi|^{2}\right\rangle=v^{2}=\mu_{0}^{2} / \lambda_{0}$ ) the same condition implies that the Higgsmass of the fermion is cancelled out. This is schematically shown in fig 2 .

It is important to note that the quadratic divergency of the loop integrals both in fig. 1 and 2 is exactly compensated by the $b^{2}$ factor coming from the insertion of the Wilson-like vertex. In the following we will encounter other instances of this UV vs. IR compensation.

\footnotetext{
${ }^{3}$ By Quantum Effective Lagrangian we mean the generating functional of the 1PI vertices of the theory.
} 


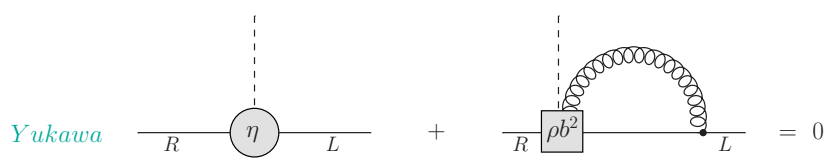

Figure 1. The condition determining $\eta_{c r}$ in the Wigner phase. The box represents the insertion of the Wilson-like vertex.

mass
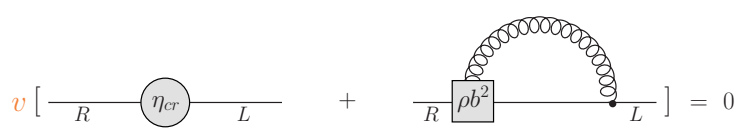

Figure 2. The condition determining $\eta_{c r}$ in the NG phase.

\subsection{NP mass generation}

Now the key observation is that at $\eta=\eta_{c r}$ where in the NG phase the fermion Higgs-mass is cancelled, the quark acquires a non-vanishing NP mass via a UV vs. IR compensation mechanism, reminiscent of the one that makes finite the 1-loop diagrams of figs. 1 and 2.

Because of this kind of subtle UV vs. IR compensations, it is crucial to analyze and determine the structure of the formally $\mathrm{O}\left(b^{2}\right)$ terms of the regularized theory. This can be properly done by making recourse to the Symanzik expansion technique [11].

This analysis was carried out in ref. [1] where it was shown that because of the occurrence of the spontaneous breaking of the (restored) $\tilde{\chi}_{L} \times \tilde{\chi}_{R}$ symmetry, triggered by residual $\mathrm{O}\left(b^{2}\right)$ chiral breaking terms, one must include in the Symanzik expansion also formally $\mathrm{O}\left(b^{2}\right) \mathrm{NP}-\mathrm{ly}$ generated operators of the form

$$
O_{6, \bar{q} q} \propto b^{2} \Lambda_{s} \alpha_{s}|\Phi|\left[\bar{q} \not{D}^{A} q\right], \quad O_{6, F F} \propto b^{2} \Lambda_{s} \alpha_{s}|\Phi|\left[F^{A} \cdot F^{A}\right] .
$$

The form of these operators is completely fixed by symmetries (in particular $\chi_{L} \times \chi_{R}$ ) and dimensional considerations. The presence of the $\Lambda_{s}$ factor signals their NP origin. The effect of the terms in eq. (10) can be elegantly taken into account by including in the calculations the new diagrams generated by the augmented Lagrangian

$$
\mathcal{L}_{\text {toy }} \rightarrow \mathcal{L}_{\text {toy }}+\Delta \mathcal{L}_{N P}^{S y m}, \quad \Delta \mathcal{L}_{N P}^{S y m}=b^{2} \Lambda_{s} \alpha_{s}|\Phi|\left[c_{F F} F^{A} \cdot F^{A}+c_{\bar{q} q} \bar{q} \mathscr{D}^{A} q\right]+\ldots
$$

A remarkable fact is that new diagrams do exist that contribute, among other correlators, to the quark self-energy. A couple of these diagrams are drawn in fig. 3. A straightforward calculation gives for the amputated zero momentum diagram in the left panel of fig. 3
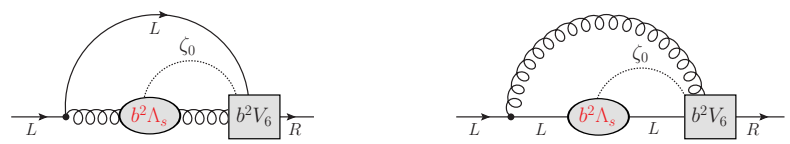

Figure 3. Two lowest loop order quark self-energy diagrams. The blobs represent insertions of $\Delta \mathcal{L}_{N P}^{S y m}$.

$$
m_{q}^{N P} \propto \alpha_{s}^{2} \int^{1 / b} \frac{d^{4} k}{k^{2}} \frac{\gamma_{\mu} k_{\mu}}{k^{2}} \int^{1 / b} \frac{d^{4} \ell}{\ell^{2}+m_{\zeta_{0}}^{2}} \frac{\gamma_{v}(k+\ell)_{v}}{(k+\ell)^{2}} \cdot b^{2} \gamma_{\rho}(k+\ell)_{\rho} b^{2} \Lambda_{s} \gamma_{\lambda}(2 k+\ell)_{\lambda} \sim \alpha_{s}^{2} \Lambda_{s} .
$$

Here again we get a finite result, owing to an exact compensation between the UV power divergencies of the 2-loop integrals and the IR behaviour determined by the expression of the Wilson-like vertex (7) and the physics of the spontaneous breaking of the chiral $\tilde{\chi}_{L} \times \tilde{\chi}_{R}$ symmetry encoded in the form of the Symanzik operators (10). A similar compensation occurs in the calculation of the diagram in the right panel of fig. 3 . 


\subsection{The QEL of the critical theory in the NG phase}

The resulting non-vanishing quark mass, the occurrence of which was checked in explicit lattice simulations of the model (3) in ref. [2], can be incorporated in the QEL, $\Gamma^{N G}$, of the theory, upon introducing the non-analytic field $U$ in the polar decomposition

$$
\Phi=\left(v+\zeta_{0}\right) U, \quad U=\exp [i \vec{\tau} \vec{\zeta} / v]
$$

As $U$ transforms like $\Phi$, new $\chi_{L} \times \chi_{R}$ invariant operators can indeed be constructed, yielding

$$
\begin{aligned}
\Gamma_{d=4}^{N G} & =\frac{1}{4}\left(F^{A} \cdot F^{A}\right)+\bar{q}_{L} \mathscr{D}^{A} q_{L}+\bar{q}_{R} \mathcal{D}^{A} q_{R}+ \\
& +c_{q} \Lambda_{s}\left[\bar{q}_{L} U q_{R}+\bar{q}_{R} U^{\dagger} q_{L}\right]+\frac{c^{2} \Lambda_{s}^{2}}{2} \operatorname{Tr}\left[\partial_{\mu} U^{\dagger} \partial_{\mu} U\right] .
\end{aligned}
$$

Naturally, of special interest is the first term in the second line of eq. (14) because, upon expanding $U=11+\vec{\tau} \vec{\zeta} / v+\ldots$, it gives raise to a mass for the fermion plus a wealth of NG boson interactions ${ }^{4}$.

\section{Introducing weak and Tera-interactions}

To proceed to the construction of a possible, realistic beyond-the-SM-model (bSMm) we clearly need to introduce EW interactions. At the same time, as mentioned in the Introduction, it is also necessary to extend the model by incorporating a super-strongly interacting sector, so that the whole theory will have an RGI scale, $\Lambda_{T}$, much larger than $\Lambda_{Q C D}$ and of the order of a few TeVs. Only in this way there is the chance that eq. (1) can yield the correct order of magnitude of the top quark mass. The extended Lagrangian is obtained by doubling the structure of quarks in order to encompass Tera-particles ( $Q=$ Tera-quarks and $G=$ Tera-gluons) and gauging the exact $\chi_{L}$ symmetry to introduce weak interactions. The whole Lagrangian will read

$$
\begin{aligned}
& \mathcal{L}(q, Q ; \Phi ; A, G, W)=\mathcal{L}_{k i n}(q, Q ; \Phi ; A, G, W)+\mathcal{V}(\Phi)+\mathcal{L}_{\text {Yuk }}(q, Q ; \Phi)+\mathcal{L}_{W i l}(q, Q ; \Phi ; A, G, W) \\
& \text { - } \mathcal{L}_{k i n}(q, Q ; \Phi ; A, W)=\frac{1}{4}\left(F^{A} \cdot F^{A}+F^{G} \cdot F^{G}+F^{W} \cdot F^{W}\right)+ \\
& +\left[\bar{q}_{L} \mathcal{D}^{A W} q_{L}+\bar{q}_{R} \mathcal{D}^{A} q_{R}\right]+\left[\bar{Q}_{L} \mathcal{D}^{A G W} Q_{L}+\bar{Q}_{R} \mathcal{D}^{A G} Q_{R}\right]+\frac{k_{b}}{2} \operatorname{Tr}\left[\left(\mathcal{D}_{\mu}^{W} \Phi\right)^{\dagger} \mathcal{D}_{\mu}^{W} \Phi\right] \\
& \text { - } \mathcal{V}(\Phi)=\frac{\mu_{0}^{2}}{2} k_{b} \operatorname{Tr}\left[\Phi^{\dagger} \Phi\right]+\frac{\lambda_{0}}{4}\left(k_{b} \operatorname{Tr}\left[\Phi^{\dagger} \Phi\right]\right)^{2} \\
& \text { - } \mathcal{L}_{Y u k}(q, Q ; \Phi)=\eta_{q}\left(\bar{q}_{L} \Phi q_{R}+\bar{q}_{R} \Phi^{\dagger} q_{L}\right)+\eta_{Q}\left(\bar{Q}_{L} \Phi Q_{R}+\bar{Q}_{R} \Phi^{\dagger} Q_{L}\right) \\
& \text { - } \mathcal{L}_{W i l}(q, Q ; \Phi ; A, G, W)=\frac{b^{2}}{2} \rho_{q}\left(\bar{q}_{L} \overleftarrow{\mathcal{D}}_{\mu}^{A W} \Phi \mathcal{D}_{\mu}^{A} q_{R}+\bar{q}_{R} \overleftarrow{\mathcal{D}}_{\mu}^{A} \Phi^{\dagger} \mathcal{D}_{\mu}^{A W} q_{L}\right)+ \\
& \quad+\frac{b^{2}}{2} \rho_{Q}\left(\bar{Q}_{L} \overleftarrow{\mathcal{D}}_{\mu}^{A G W} \Phi \mathcal{D}_{\mu}^{A G} Q_{R}+\bar{Q}_{R} \overleftarrow{\mathcal{D}}_{\mu}^{A G} \Phi^{\dagger} \mathcal{D}_{\mu}^{A G W} Q_{L}\right)
\end{aligned}
$$

with obvious notations for the covariant derivatives. Besides the Yukawa (eq. (18)) and the Wilson-like (eq. (19)) operators, now also the kinetic term of the scalar (last term in eq. (16)) breaks $\tilde{\chi}_{L} \times \tilde{\chi}_{R}$ and mixes with $\mathcal{L}_{Y u k}$ and $\mathcal{L}_{W i l}$. Thus to get to the critical theory (invariant under $\tilde{\chi}_{L} \times \tilde{\chi}_{R}$ ), on top of $\eta_{q}$ and $\eta_{Q}$, a further parameter, $k_{b}$, needs to be introduced and appropriately tuned. The conditions determining the critical theory correspond to have vanishing effective Yukawa interactions and vanishing scalar kinetic term in the QEL. Notice that the coefficient $k_{b}$ appears also in the expression of the scalar potential (17). The reason for this is that in this

\footnotetext{
${ }^{4}$ We may notice that in the NP mass, $U$ has a role similar to the one the non-analytic $r /|r|$ ratio has in WLQCD, as if we had promoted the Wilson $r$ parameter to a field, $\Phi$.
} 
way the (bare) $\mathrm{vev}^{2}$ (in the NG phase) and the quartic coupling of the canonically normalized scalar field will have the standard definitions, $v^{2}=\left|\mu_{0}^{2}\right| / \lambda_{0}$ and $\lambda_{0}$.

The tuning conditions that determine $\eta_{q c r}, \eta_{Q c r}$ and $k_{b c r}$ in the Wigner phase are schematically reported in the figs. 4, 5 and 6 . To the lowest loop order one finds

$$
\begin{gathered}
\eta_{q \text { cr }}^{(1-\text { loop })}=\rho_{q} \eta_{1 q} \alpha_{s}, \quad \eta_{Q c r}^{(1-\text { loop })}=\rho_{Q} \eta_{1 Q} \alpha_{T}, \\
k_{b c r}^{(1-\text { loop })}=\left[\rho_{q}^{2} N_{c}+\rho_{Q}^{2} N_{c} N_{T}\right] k_{1},
\end{gathered}
$$

where $\eta_{1 q}, \eta_{1 Q}$ and $k_{1}$ are computable coefficients and $\mathrm{SU}\left(N_{T}\right)$ is the Tera-gauge group. As before, UV loop divergencies are exactly compensated by the IR behaviour of the inserted Wilson-like vertices.
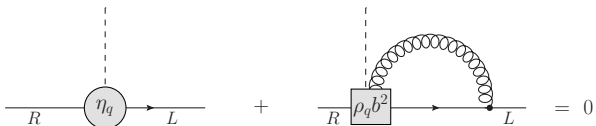

Figure 4. The tuning condition determining $\eta_{q c r}$ at lowest order. The box represents the insertion of the quark Wilson-like vertex.
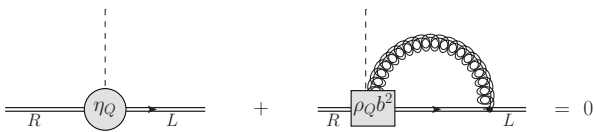

Figure 5. The tuning condition determining $\eta_{Q c r}$ at lowest order. Double lines represent Tera-particles. The box represents the insertion of the Tera-quark Wilson-like vertex.

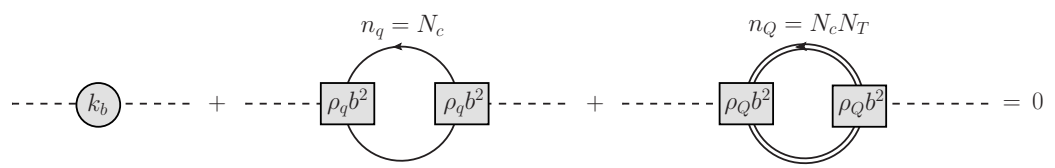

Figure 6. The tuning condition determining $k_{b c r}$ at lowest order. Notations are as in figs. 4 and 5 .

In the NG phase the above tuning conditions imply the cancellation of the Higgs-like mass of quarks, Tera-quarks and $W$ 's, as schematically represented in the figs. 7, 8 and 9.
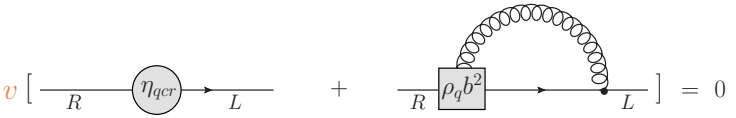

Figure 7. The mechanism behind the cancellation of the Higgs-like quark mass term, $v \bar{q} q$, operating in the NG phase of the critical theory.
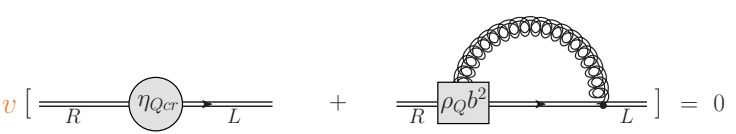

Figure 8. The mechanism behind the cancellation of the Higgs-like Tera-quark mass term, $v \bar{Q} Q$, operating in the NG phase of the critical theory.

Extending to the present case the Symanzik analysis of the formally $\mathrm{O}\left(b^{2}\right)$ operators necessary to describe the NP effects related to the spontaneous breaking of the (recovered) $\tilde{\chi}_{L} \times \tilde{\chi}_{R}$ symmetry in the regularized theory, one finds the following set of operators

$$
\begin{gathered}
\bullet O_{6, \bar{Q} Q}^{T}=b^{2} \alpha_{T} \rho_{Q} \Lambda_{T}|\Phi|\left[\bar{Q}_{L} \mathcal{D}^{A G W} Q_{L}+\bar{Q}_{R} \mathcal{D}^{A G} Q_{R}\right] \\
\bullet O_{6, \bar{Q} Q}^{s}=b^{2} \alpha_{s} \rho_{Q} \Lambda_{T}|\Phi|\left[\bar{Q}_{L} \mathcal{D}^{A G W} Q_{L}+\bar{Q}_{R} \mathcal{D}^{A G} Q_{R}\right] \\
\bullet O_{6, G G}=b^{2} \alpha_{T} \rho_{Q} \Lambda_{T}|\Phi| F^{G} \cdot F^{G} \quad \bullet O_{6, A A}=b^{2} \alpha_{s} \rho_{Q} \Lambda_{T}|\Phi| F^{A} \cdot F^{A}
\end{gathered}
$$




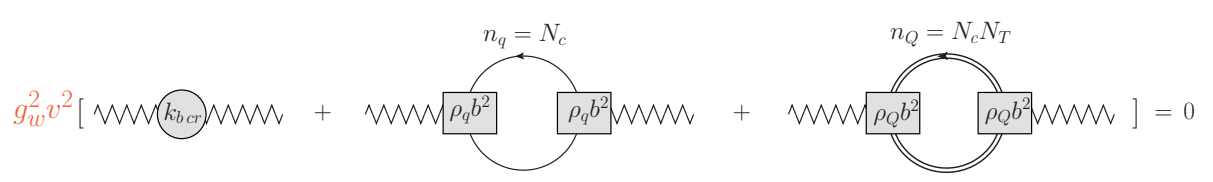

Figure 9. The mechanism behind the cancellation of the Higgs-like $W$ mass term, $g_{w}^{2} v^{2} \operatorname{Tr}\left[W_{\mu} W_{\mu}\right]$, operating in the NG phase of the critical theory.

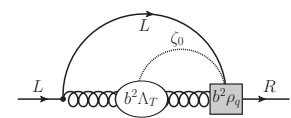

quark
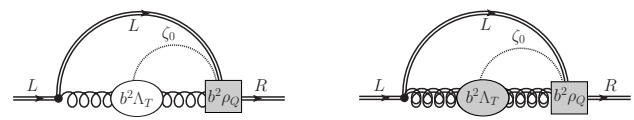

Tera-quark

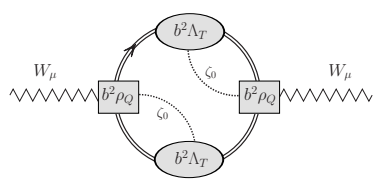

W

Figure 10. Examples of self-energy diagrams giving NP masses to quarks, Tera-quarks and $W$. The blobs represent the insertion of the NP Symanzik operators of eqs. (22), (23) and (24),

NP masses emerge from the kind of diagrams shown in fig. 10. They are all finite owing to the by now well-known UV-IR compensation and all of $\mathrm{O}\left(\Lambda_{T}\right)$ times gauge coupling dependent coefficient functions (see next section).

\subsection{The critical QEL in the NG phase}

With the same line of arguments we used before to derive eq. (14), we get for the $d=4$ piece of the QEL of the critical theory in the NG phase the expression

$$
\begin{aligned}
& \Gamma_{4 c r}^{N G}(q, Q ; \Phi ; A, G, W)=\frac{1}{4}\left(F^{A} \cdot F^{A}+F^{G} \cdot F^{G}+F^{W} \cdot F^{W}\right)+ \\
& \quad+\left[\bar{q}_{L} \mathcal{D}^{W A} q_{L}+\bar{q}_{R} \mathcal{D}^{A} q_{R}\right]+C_{q} \Lambda_{T}\left(\bar{q}_{L} U q_{R}+\bar{q}_{R} U^{\dagger} q_{L}\right)+ \\
& \quad+\left[\bar{Q}_{L} \mathscr{D}^{W A G} Q_{L}+\bar{Q}_{R} \mathcal{D}^{A G} Q_{R}\right]+C_{Q} \Lambda_{T}\left(\bar{Q}_{L} U Q_{R}+\bar{Q}_{R} U^{\dagger} Q_{L}\right)+\frac{1}{2} c_{w}^{2} \Lambda_{T}^{2} \operatorname{Tr}\left[\left(\mathcal{D}_{\mu}^{W} U\right)^{\dagger} \mathcal{D}_{\mu}^{W} U\right] .
\end{aligned}
$$

From a detailed analysis of the diagrams in fig. 10 one can read-off the parametric dependence of the mass of quarks, Tera-quarks and $W$ s to the leading loop order. One finds

$$
\begin{array}{ll}
m_{q}^{N P}=C_{q} \Lambda_{T}, & C_{q}=\mathrm{O}\left(\alpha_{s}^{2}\right) \\
m_{Q}^{N P}=C_{Q} \Lambda_{T}, & C_{Q}=\mathrm{O}\left(\alpha_{T}^{2}, \ldots\right) \\
M_{W}^{N P}=C_{w} \Lambda_{T}, & C_{w}=\sqrt{\alpha_{w}} c_{w}, c_{w}=k_{w} \mathrm{O}\left(\alpha_{T}, \ldots\right) .
\end{array}
$$

\section{Universality}

A key point that needs to be thoroughly discussed is to what extent the NP mass predictions we have derived above are "universal", or in other words to what extent their value depends on the exact form of the $d>4$ Wilson-like terms that one decides to introduces in the fundamental Lagrangian. Actually it turns out that the power of the gauge coupling multiplying 
the RGI in the leading loop order in the formula expressing the NP masses may depend on the dimensions of such chiral breaking terms. For instance, one can see that generically the higher is the dimension of the Wilson-like terms the larger will be the power of the gauge couplings in front of the RGI scale in the mass formulae ${ }^{5}$.

This may appear as a blunt violation of universality, however, one can imagine to exploit this feature to try to interpret family mass hierarchy (from heavy to light) as due to Wilsonlike terms of increasing dimensions. Implementing this idea is not at all trivial, if we want to get flavour as an emerging quantum number that identifies different families, somehow related to the "geometry" of the Wilson-like terms.

\section{Conclusions and Outlook}

We have shown that, as an alternative to the Higgs mechanism, masses can be NP-ly generated in a strongly interacting theory where chiral symmetry, broken at the UV cutoff level by irrelevant Wilson-like terms, is recovered at low energy owing to the tuning of certain Lagrangian parameters. Since an exact symmetry prevents the existence of standard mass terms of the kind $m \bar{\psi} \psi$ and there is no Higgs field around, the mechanism we are advocating here allows a neat solution of the naturalness problem. In fact, no power divergencies can affect physical masses and masses are "small", i.e. $\mathrm{O}\left(\Lambda_{\mathrm{RGI}}\right)$, as the massless theory enjoys an enhanced (chiral) symmetry, consistently with the 't Hooft idea of naturalness [3].

We have seen that to cope with the magnitude of the $W$ and top mass, a super-interacting sector, gauge invariantly coupled to standard matter, must exists leading to a theory with an RGI scale $\Lambda_{\text {RGI }}=\Lambda_{T}$ of the order of a few TeVs. The simplest model discussed in ref. [1] enjoying the NP mass generation mechanism can be straightforwardly extended to incorporate weak interactions and the new Tera-degrees of freedom. In a similar way one could introduce leptons and the hypercharge interaction, an issue that we defer to a forthcoming investigation.

\section{Acknowledgements}

We thank R. Frezzotti for infinitely many discussions on the issues presented in this talk. We would also like to acknowledge some exchange of ideas with M. Garofalo.

\section{References}

[1] R. Frezzotti and G. C. Rossi, Phys. Rev. D 92 (2015) no.5, 054505.

[2] S. Capitani et al., Phys. Rev. Lett. 123 (2019) no.6, 061802.

[3] G. 't Hooft, "Naturalness, Chiral Symmetry and Spontaneous Chiral Symmetry Breaking" in "Recent Developments in Gauge Theories" (Plenum Press, 1980).

[4] L. Susskind, Phys. Rev. D 20 (1979) 2619.

[5] S. Weinberg, Phys. Rev. D 19 (1979) 1277.

[6] S. L. Glashow, [arXiv:0504287 [hep-ph]].

[7] R. Frezzotti and G. C. Rossi, PoS LATTICE 2018 (2018) 190.

[8] R. Frezzotti and G. Rossi, in Strong dynamics for physics within and beyond the Standard Model at LHC and Future Colliders (LFC19) 9-13 September 2019, Trento, Italy.

[9] R. Frezzotti, M. Garofalo and G. C. Rossi Phys. Rev. D 93 (2016) no.10, 105030.

[10] R. Frezzotti and G. C. Rossi, Pos(Lattice2013) 354 (2013), [arXiv:1312.1069 [hep-lat]].

[11] K. Symanzik, Nucl. Phys. B 226 (1983) 187 and 205.

[12] M. Bochicchio, L. Maiani, G. Martinelli, G. Rossi, M. Testa, Nucl. Phys. B262 (1985) 331.

\footnotetext{
${ }^{5}$ As for the $\rho$ dependence of the NP masses, one can prove that in the case of a theory with only quarks and $W$ 's (and not Tera-quarks or leptons) their mass is actually $\rho$ independent. If other fermions are present, be them Tera-particles or leptons, NP masses will be functions of the ratios of the $\rho$ parameters of the various fermions.
} 